\title{
Protocol
}

\section{Efficacy of colchicine in moderately symptomatic COVID-19 patients: a study protocol for a double-blind, randomized, placebo-controlled trial}

\author{
Motlabur Rahman, Mujibur Rahman, Ponkaj K. Datta*, Khairul Islam, Pratyay Hasan, \\ Manjurul Haque, Md Imtiaz Faruq, Mohiuddin Sharif, Rifat H. Ratul
}

Department of Medicine, Dhaka Medical College, Dhaka, Bangladesh

Received: 12 October 2020

Accepted: 29 December 2020

*Correspondence:

Dr. Ponkaj K. Datta,

E-mail: ponkajdatta@yahoo.com

Copyright: (c) the author(s), publisher and licensee Medip Academy. This is an open-access article distributed under the terms of the Creative Commons Attribution Non-Commercial License, which permits unrestricted non-commercial use, distribution, and reproduction in any medium, provided the original work is properly cited.

\section{ABSTRACT}

Background: Inflammation is playing a major role in the pathophysiology of severe COVID-19 disease. The main causes of mortality are cytokine syndrome and immune thromboembolism. Colchicine is an anti-inflammatory drug but its action is mediated by completely different pathophysiologic routes than that of corticosteroids and non-steroidal anti-inflammatory agents. Colchicine inhibits neutrophil chemotaxis, inhibits inflammasome signaling and reduces interleukin-1 $\beta$, reduces neutrophil-platelet interaction and aggregation. Colchicine is a readily available, cheap drug, has been used safely for many years. Specific targeted anti-inflammatory drugs like tocilizuma and anakinra are costly. A previous study suggested a significant clinical benefit from colchicine in patients hospitalized with COVID-19. But they did not compare with placebo. So, we have designed this study.

Methods: This is a prospective, double-blind, randomized, placebo-controlled clinical trial. The study will be conducted at Dhaka medical college hospital, Bangladesh. Real time-polymerase chain reaction (RT-PCR) positive COVID-19 patients with moderate symptoms will be included in this study. Participants will be randomized into two groups at $1: 1$ ratio. Patients of one group will be treated with standard treatment along with colchicine for 14 days. The patients in other group will be treated with standard treatment along with placebo for the same duration. The primary outcome of the study will be time to develop clinical deterioration, defined as the time from randomization to a deterioration of two points (from the status at randomization) on a seven-category ordinal scale.

Conclusions: Enrolment of participants has begun at the study site. A total of 300 participants will be enrolled.

Trial Registration: ClinicalTrials.gov identifier: NCT04527562.

Keywords: Anti-inflammatory agents, Colchicine, COVID-19

\section{INTRODUCTION}

A deadly pandemic of COVID- 19 has been started from Wuhan, China in early December 2019. So far 215 countries, areas or territories already involved throughout the glove leaving 5934936 patients infected and 367166 killed. Bangladesh is also affected since its first case detection on 08 April 2020.
Up to 31 May 2020, the total number of the positive case was 44608 with 610 deaths. ${ }^{1}$ As the SARS-CoV-2 making its way around the world, researchers and doctors are in search of drugs to treat and stop the spread of the disease. Since there are no specific therapeutic options available at present, health officials are primarily relying on quarantining the infected to contain the virus spread and repurposing already existing anti-viral drugs and antibiotics to treat infection based on symptoms. ${ }^{2}$ 


\section{Background}

The disease caused by the novel SARS-CoV-2 is named COVID-19 by World Health Organization (WHO). The culprit virus belongs to the coronaviridea family of corona viruses that caused two other outbreaks, namely severe acute respiratory syndrome (SARS) in 2002 and Middle East respiratory syndrome (MERS) in 2012. ${ }^{3}$ SARS-CoV2 is a single-stranded, positive strain ribonucleic (RNA) virus with a protein shell and membrane. Due to their dependency on hosts' molecular machinery to replicate themselves, these RNA based viruses are prone to mutations when they transfer from one animal to another. When infection transfers from animal to human, they can evolve into a new virus like SARS-CoV, MERS-CoV and SARS-CoV-2. The genetic information of the virus is the starting point in understanding the origin of the pathogen and one of the valuable information in designing strategies to fight against it. As soon as the genetic makeup of SARSCoV-2 is revealed by Ren et al on 25 January 2020, researchers across the globe began comparing the novel coronavirus's genome with SARS and MERS to determine if any of the drugs developed against SARS and MERS can work against this novel SARS-CoV-2.,5

Based on ongoing epidemiological investigations, the incubation period of the virus is 1-14 days, mostly 3-7 days. SARS-CoV-2 is contagious during the latency period. ${ }^{6,7}$ Based on the knowledge acquired during these months the infection has been divided into three phases, the first asymptomatic or with mild symptoms, the second and third characterized by hyperactive inflammatory state responsible for lung lesions and that in some patients can rapidly develop acute respiratory distress syndrome, respiratory failure, multiple organ failure, and even death. Phase two and three of the infection is characterized by a hyperactive inflammatory state that can result in a cytokine storm (CS). ${ }^{8}$ Sudden and rapidly progressing clinical deterioration has been widely mentioned in late stages of COVID-19 (around 7-10 days). ${ }^{9,10}$ This often manifests as an unexpected aggravation of symptoms (fever, dyspnea) and is correlated with increased levels of acute-phase reactants (ESR, CRP, ferritin), coagulopathy (elevated titers of d-dimers, disseminated intravascular coagulation), and cell lysis (CK, LDH). In the most severe patients, clinical and laboratory parameters correlated with increased levels of proinflammatory cytokines (IL-1 $\beta$, IL1Ra, IL-6, TNF- $\alpha$, and sIL2-R $\alpha$ ), evocative of a cytokine storm. Interestingly, ARDS occurs in SARS-CoV patients despite a diminishing viral load, suggesting that exuberant host immune response may be responsible for this outcome rather than viral virulence. Such a cytokine profile is strongly reminiscent of both cytokine release syndrome (CRS, seen in CAR T cell therapy) and hemophagocytic lymphohistiocytosis (HLH). Numerous authors have paralleled the COVID-19 cytokine storm to either primary or reactive HLH (reHLH) because of its close resemblance, including high fever, cytopenia, hyperferritinemia, abnormal liver tests, coagulopathy, and pulmonary involvement (including ARDS), occurring in approximately $50 \%$ of patients with reHLH. In adults, reHLH is most often triggered by viral infections and is observed in $3-4 \%$ of sepsis cases. Herpesviridae (e.g. Epstein-Barr virus) and influenza are major triggers of such cytokine storms. Systemic diseases, like systemic lupus erythematosus, or the autoinflammatory adult-onset Still's disease and its pediatric counterpart can also be complicated by cytokine storm, known as the macrophage activation syndrome (identical to reHLH). In all these conditions, IL-1 $\beta$, IL-18, IFN- $\gamma$, and IL-6 are key mediators of hyperinflammation. Analyzing more precisely the immunopathology of SARS-CoV2-related ARDS, Giamarellos-Bourboulis et al have concluded that two patterns of immune dysfunction exist in worsening COVID-19: one pattern highly suggestive of macrophage activation syndrome (hyperferritinemia and elevated $\mathrm{H}$ score: $25 \%$ of patients), which is driven by IL- $1 \beta$; and one pattern with immune dysregulation driven by IL-6. The latter was characterized by a combination of hypercytokinemia, immunoparalysis (as indicated by decreased HLA-DR molecules on CD14 monocytes), and global lymphopenia (including CD4+ and NK cells). Interestingly, IL-6 blockade with tocilizumab partially restored HLA-DR expression on CD14 monocytes and increased the circulating lymphocyte count. ${ }^{10,11}$

Based on the inflammatory nature of the severe COVID19 disease many anti-inflammatory drugs have been studied with variable efficacy. They include targeted antiinflammatory therapy e.g. IL 1, IL 6 inhibitors, interferongamma, TNF alfa inhibitors, and non-targeted antiinflammatory e.g. corticosteroid, hydroxychloroquine, JAK inhibitors and colchicine. ${ }^{11-13}$ Among them colchicine is a long-established drug with anti-inflammatory properties used to treat patients with Behçet's disease or familial Mediterranean fever. Colchicine inhibits IL-1 $\beta$ and its subsequent inflammatory cascade principally by blocking pyrin and (to a lesser extent) NLRP3 inflammasome activation. Until now, there has been no data indicating that pyrin is activated upon SARS-CoV-2 infection and this would appear rather unlikely. On the other hand, NLRP3 is likely to be activated following virus entry into the cell. Nevertheless, the inhibition of NLRP3 inflammasome by colchicine has not been firmly demonstrated since in vitro it does not prevent IL $1 \beta$ secretion induced by typical NLRP3 stimuli (i.e. ATP, nigericin). However, five RCTs are currently underway to test the efficacy of colchicine in COVID-19 patients (NCT04322682, NCT04322565, NCT04328480, NCT04326790, NCT04350320). ${ }^{11,14}$

\section{Rationale}

In Bangladesh colchicine is a readily available drug, has been used for many years for established indications. It is a cheap drug. Specific targeted anti-inflammatory drugs like tocilizuma and anakinra is costly. As a lower middle income country, these drugs will not be affordable to our people as well as it will be a burden for the government. If colchicine is found to be effective in the treatment of 
COVID-19 it can be recommended in our national guidelines. A previous study suggested a significant clinical benefit from colchicine in patients hospitalized with COVID-19. They found reduced severe disease and mortality in colchicine treated patients, associated with lower d-Dimer level than control. But they did not compare with placebo. So, we have designed this doubleblind placebo-controlled study. ${ }^{15}$

\section{METHODS}

\section{Trial design}

This is a prospective, double-blind, randomized, placebocontrolled clinical trial. We will randomize the patients into two groups. Patients of one group will be treatment group. They will be treated with optimal treatment based on the algorithm proposed in national guidelines on clinical management of coronavirus disease 2019 (COVID-19) Version 7.0, 28 May 2020, disease control division, Directorate General of Health Services, Ministry of Health and Family Welfare, Government of the People's Republic of Bangladesh along with colchicine. ${ }^{16}$ The patients in other group will be controlled group. They will be treated with optimal treatment based on the algorithm proposed in national guideline along with a placebo.

\section{Study setting}

The study will be conducted at Dhaka Medical College Hospital. It is the leading tertiary level teaching hospital located at the capital city of Bangladesh. The hospital has a 600 bed COVID-19 dedicated unit with a high dependency unit, intensive care unit, and coronary care unit and dialysis unit facilities.

\section{Eligibility criteria}

\section{Study population}

Patients with laboratory confirmation of COVID-19 infection with clinical signs and symptoms of the moderate disease are our study population.

\section{Selection criteria}

Competent males and females of at least 18 years of age, can swallow tablets, RT-PCR for SARS CoV-2 positive within the last 3 days, having moderate symptoms that include fever or history of fever, cough and/or shortness of breath, oxygen saturation $94 \%$ or more, pulmonary ground-glass opacity or consolidations on chest imaging (chest x-ray or CT scan of the chest) involving less than $50 \%$ area of lungs, CRB 65 score 0 , will be included in this study. Pregnant and lactating mothers, patients with known hypersensitivity to colchicine, known chronic illness e.g. hepatic failure, chronic kidney disease (eGFR<30 ml/min), decompensated heart failure, long QT syndrome (QTc >450 $\mathrm{msec}$ ), patient with inflammatory bowel disease, chronic diarrhea or malabsorption, the patient currently taking colchicine for other indications (mainly chronic indications represented by familial mediterranean fever or gout), the patient undergoing chemotherapy for cancer will be excluded from the study.

\section{Interventions}

\section{The explanation for the choice of comparators}

The participants randomized to the treatment group will be given tablet colchicine and standard care. Based on the inflammatory nature of the severe COVID-19 disease many anti-inflammatory drugs have been studied with variable efficacy. Among them, colchicine is a longestablished drug with anti-inflammatory properties used to treat patients with Behçet's disease or familial Mediterranean fever. Colchicine inhibits IL-1 $\beta$ and its subsequent inflammatory cascade principally by blocking pyrin and (to a lesser extent) NLRP3 inflammasome activation. In Bangladesh, colchicine is a readily available drug, has been used for many years safely for established indications. It is a cheap drug. A previous study suggested a significant clinical benefit from colchicine in patients hospitalized with COVID-19. They found less development of severe disease and mortality in colchicine treated patients, associated with lower d-Dimer level than control. But they did not compare with placebo. So, we have designed this double-blind placebo-controlled study. ${ }^{15}$

\section{Intervention description}

Active comparator/treatment group: drug: colchicine. Participants in this group will be given a starting dose of $1.2 \mathrm{mg}$ of colchicine ( 2 tablets of $0.6 \mathrm{mg}$ ) single or 12 hourly divided doses. After that, they will take colchicine $0.6 \mathrm{mg}$ daily for 13 days. If they develop gastrointestinal side effects e.g. abdominal pain, burning, vomiting, diarrhea, omeprazole and antiemetic will be prescribed. Supportive care and treatment will also be given. Placebo comparator/control group: drug: placebo. Participants in this group will be given a starting dose of 2 tablets of placebo, single or 12 hourly divided doses. After that, they will take one placebo tablet daily for 13 days. The placebo tablet will be identical to the colchicine tablet. If patients develop gastrointestinal side effects e.g. abdominal pain, burning, vomiting, diarrhea, omeprazole and antiemetic will be prescribed. Supportive care and treatment will also be given. Standard care of enrolled study patients will consist of isolation facility, symptomatic treatment with paracetamol, fexofenadine, steam inhalation/gurgle of lukewarm water, ensuring of hand wash (20 seconds each time) and wearing mask and monitoring by the attending nurses.

\section{Criteria for discontinuing or modifying allocated interventions}

All participants will preserve the right to withdraw him or herself from the study at any time and will get the usual 
medications as per decision of the consultant. Investigators can also decide to discontinue the study medications at any time for the safety of the participants. A patient may be withdrawn from the study if any serious adverse event (SAE) occurs which, in the principal investigators' judgment, requires withdrawal of the subject from the study. This may include drug intolerance or an unacceptable $\mathrm{AE}$; regardless of whether the $\mathrm{AE}$ is considered likely related to the study drug or not; significant results of laboratory tests which may require the withdrawal of the participant. Any suggestions from DSMB to the investigators to discontinue or modify the allocation of a participant for safety purpose may also play role in participant withdrawal from the study.

\section{Relevant concomitant care permitted or prohibited during the trial}

Along with study medicine, the following standard care will be given to all participants-isolation facility, symptomatic treatment with paracetamol, fexofenadine, steam inhalation/gurgle of lukewarm water, ensuring of hand wash (20 seconds each time) and wearing mask and monitoring by the attending nurses. If the participant's clinical condition deteriorates at any time than any other drug may be given as prescribed by the treating consultant.

\section{Provisions for post-trial care}

The patient will be advised to come for regular follow up at the post-acute COVID-19 clinic on day 28 and subsequently if needed. This clinic is run by the study institution.

\section{Study outcomes}

Primary outcome name: Time to develop clinical deterioration, defined as the time from randomization to a deterioration of two points (from the status at randomization) on a seven-category ordinal scale. Metric/method of measurement: seven-category ordinal scale. The scale is recommended by the WHO R\&D Blueprint expert group. The seven-category ordinal scale consisted of the following categories: not hospitalized with the resumption of normal activities; not hospitalized, but unable to resume normal activities; hospitalized, not requiring supplemental oxygen; hospitalized, requiring supplemental oxygen; hospitalized, requiring nasal highflow oxygen therapy, non-invasive mechanical ventilation, or both; hospitalized, requiring ECMO, invasive mechanical ventilation, or both; and death. ${ }^{17-19}$ Timepoint is 14 days following randomization.

Secondary outcome names: length of hospital stay, proportions of participant requiring an increased amount of supplemental oxygen, proportions of participants requiring mechanical ventilation, and proportions of allcause mortality among the participants. Time frame for all is 14 days post-randomization.

\section{Sample size}

\section{Sampling}

Simple random sampling (lottery) method was used.

\section{Sample size with the statistical basis}

Total sample size was 300, sample size in colchicine group was 150, and sample size in the control group was 150 .

This double-blinded, placebo-controlled trial proposes to assess the effectiveness of colchicine in curing patients suffering from COVID-19 in comparison to placebo. We assume that the proportion of subjects will be cured by colchicine is $50 \%$ and a clinically important difference of $16 \%$ as compared to placebo is acceptable.

The level of significance was $5 \%$, power was $80 \%$, and type of test was two-sided.

Formula of calculating sample size is. ${ }^{20}$

$$
\begin{gathered}
n=[(Z \alpha / 2+Z \beta) 2 \times\{(p 1(1-p 1)+(p 2(1 \\
-p 2))\}] /(p 1-p 2) 2
\end{gathered}
$$

Where

$n=$ sample size required in each group,

$p 1=$ proportion of subject cured by colchicine $=0.50$,

$p 2=$ proportion of subject cured by placebo $=0.34$,

$p 1-p 2=$ clinically significant difference $=0.16$

$Z \alpha / 2=$ depends on level of significance, for $5 \%$ this is 1.96

$Z \beta=$ depends on power, for $80 \%$ this is 0.84

Based on above formula the sample size required per group is 146 . Hence total sample size required is 292 .

So, a sample size of 292 patients, 146 in each arm, is sufficient to detect a clinically important difference of $16 \%$ between groups in curing COVID-19 using a two-tailed ztest of proportions between two groups with $80 \%$ power and a $5 \%$ level of significance. This $16 \%$ difference represents a $50 \%$ cure rate using colchicine and a $34 \%$ cure rate using standard treatment and placebo.

For convenience, we will recruit a total of 300 patients, 150 patients in the colchicine treatment group and 150 patients in the control group (placebo group). So, sample size in colchicine group is 150 and sample size in the control group is 150 . 
Table 1: Study timeline of participant assessments/interventions.

\begin{tabular}{|c|c|c|c|c|c|c|}
\hline \multirow{3}{*}{$\begin{array}{l}\text { Assessments/interventions } \\
\text { Timepoint }\end{array}$} & \multirow{2}{*}{$\begin{array}{l}\text { Study period } \\
\text { Screening/bas- } \\
\text { eline/randomiz } \\
\text {-ation }\end{array}$} & \multirow{3}{*}{$\begin{array}{l}\text { Treat- } \\
\text { ment } \\
\text { phase } \\
\text { D1- } \\
\text { D14 }\end{array}$} & \multirow{2}{*}{\multicolumn{3}{|c|}{ Follow-up phase }} & \multirow{3}{*}{$\begin{array}{l}\text { Data } \\
\text { processing } \\
\text { and analysis } \\
\text { Up to } 6 \text { months }\end{array}$} \\
\hline & & & & & & \\
\hline & D0 & & $\begin{array}{lll}\mathrm{D} & \mathrm{D} & \mathrm{D} \\
3 & 7 & 10\end{array}$ & $\begin{array}{l}\mathrm{D} \\
14\end{array}$ & $\begin{array}{l}\mathrm{D} \\
21\end{array}$ & \\
\hline Enrolment & & & & & & \\
\hline Inclusion/exclusion criteria & $\mathrm{X}$ & & & & & \\
\hline Written informed consent & $\mathrm{X}$ & & & & & \\
\hline Demographics & $\mathrm{X}$ & & & & & \\
\hline Assessment of concomitant chronic diseases & $\mathrm{X}$ & & & & & \\
\hline Efficacy/safety assessment & $\mathrm{X}$ & $\mathrm{X}$ & $\mathrm{X} X \mathrm{X}$ & $\mathrm{X}$ & $\mathrm{X}$ & \\
\hline ECG & $\mathrm{X}$ & & & & & \\
\hline Chest imaging & $\mathrm{X}$ & & & & & \\
\hline Vital signs & $\mathrm{X}$ & $\mathrm{X}$ & & & & \\
\hline Body temperature, oxygen saturation & $\mathrm{X}$ & $\mathrm{X}$ & $\mathrm{X} \quad \mathrm{X} \quad \mathrm{X}$ & $\mathrm{X}$ & & \\
\hline Clinical symptoms assessment & $\mathrm{X}$ & & & & & \\
\hline Urine for pregnancy test & $\mathrm{X}$ & & & & & \\
\hline Fever, cough, diarrhea, dyspnea & $\mathrm{X}$ & $\mathrm{X}$ & $\mathrm{X} X \mathrm{X}$ & $\mathrm{X}$ & $\mathrm{X}$ & \\
\hline Interventions & & & & & & \\
\hline Colchicine/placebo & & $\mathrm{X}$ & & & & \\
\hline $\begin{array}{l}\text { Concomitant medications assessment } \\
\text { Lab assessments }\end{array}$ & & & $\mathrm{X} \quad \mathrm{X} \quad \mathrm{X}$ & $\mathrm{X}$ & & \\
\hline Whole blood count (sampling time) & $\mathrm{X}$ & & $\mathrm{X} X \mathrm{X}$ & $\mathrm{X}$ & & \\
\hline Serum creatinine, SGPT, RBS & $\mathrm{X}$ & & $\mathrm{X}$ & $\mathrm{X}$ & & \\
\hline CRP, Serum ferritin, d-Dimer, LDH & & & $\mathrm{X} \times \mathrm{X}$ & $\mathrm{X}$ & & \\
\hline $\begin{array}{l}\text { Nasopharyngeal swab for RT-PCR for } \\
\text { SARS-CoV-2 (up to } 2 \text { negative) }\end{array}$ & & & $\mathrm{X} X \mathrm{X}$ & $\mathrm{X}$ & & \\
\hline
\end{tabular}

\section{Recruitment}

All RT-PCR positive patients admitted to the study site are our study population. From them, sample will be collected based on inclusion and exclusion criteria. Participants will be properly informed and written consent will be taken. No advertisement for participant enrolment will be done.

\section{Assignment of interventions: allocation}

\section{Sequence generation}

Three hundred identical opaque envelopes will be prepared by an independent statistician. He will mark them from 1 to 300. Each envelope will contain either a full course of drugs or placebo for one patient. Envelops will be filled randomly and statistician will keep a record of its content in a secured place. Colchicine and placebo blisters within each envelope are identical in size and labeling. Sequencing will be done manually by the lottery. The statistician will also supply 300 identical cards containing numbers 1 to 300. After signing informed consent each participant will take a card randomly by lottery. That number will be assigned to the participant as his ID number. The study nurse will supply the sealed envelope with the same number containing either drug or placebo.

\section{Concealment mechanism}

Identical opaque sealed envelopes will be prepared by an independent statistician. The information about the content of each envelop will be kept secured by the statistician. No investigator or study personnel will have access to that. Colchicine and placebo tablets will have the same size, shape, colour and smell. The colour, size, shape of the blister and the labeling will be the same. Investigators will know the number of the envelope but not the content.

\section{Assignment of interventions: blinding}

\section{Who will be blinded?}

This study is a double-blind clinical trial. The study participants, investigators, care providers, outcome assessors, and data analysts are all blinded. Treatment allocation will only be decoded after complete data analysis.

\section{Procedure for unblinding if needed}

Unblinding is permissible when investigators believe that there is a very strong need to know the study drug allocation to perform any specific treatment/ action for the safety of the participant. Whenever possible, unblinding 
will only be conducted after discussion with the study principal investigator. Unblinding and the situation lead to it will be reported to the ethical review committee. The procedure for revealing a participant's allocated intervention is as follows: investigators confirm that the patient meets the criteria of unblinding according to the protocol; the independent physician not related to the study will open the sealed envelope and inform the investigators of the allocation; information about the date, time, and reason of unblinding will be recorded in the envelope; and the envelope must be sealed again as soon as possible and securely stored.

\section{Data collection and management}

\section{Plans for assessment and collection of outcomes}

Investigators are responsible for the assessment and collection of the outcome, baseline, and other trial data, and the data will be reviewed by the study chair. All investigators will be trained about any protocol related update. Laboratory tests will be done by the standard laboratory medicine department of this hospital. The nasopharyngeal swab will be sent to a laboratory at the virology department of this medical college, where tests will be performed according to laboratory standard operating procedures (SOPs).

Plans to promote participant retention and complete follow-up

The participants will preserve the right to withdraw his name from the study. This decision must be reviewed by investigators. The investigators will explain to these participants the importance of staying in the study for the full duration of the follow-up of this trial. For any withdrawal from this trial, investigators must keep a record of withdrawal reason in the case record form (CRF) and try to complete all remaining assessments up to day 21 after randomization. For subjects who withdraw due to AEs, investigators should closely follow up their AEs until the AEs disappear, return to the baseline state, or the $\mathrm{AE}$ condition is stable.

If subjects are lost to follow-up, existing data collected up until the time of loss to follow-up will be used. Investigators may request for subjects to be withdrawn from receiving the study drugs for clinical or other reasons. In this case, subjects should remain in the trial after terminating the trial treatment and be followed up for all scheduled visits and the corresponding data recorded.

\section{Data management}

The data of each participant will be recorded into the CRF rapidly, completely, and accurately. Each completed CRF will be reviewed by the investigators. The study chair will recheck the data and ask other investigators to resolve any queries identified. He will proofread and modify the data according to investigators' answers. It is the responsibility of the principal investigator to securely store all printed and digital data.

\section{Confidentiality}

Each participant will be issued a serial number before enrollment. After obtaining informed written consent a unique ID will be issued by lottery, which will be written on the CRF. A coding chart will contain the patient name and identification which will be kept protected by the principal investigator. All communication like sending the sample to the laboratory will contain ID numbers only. All the participants' confidentiality will be maintained before, during and after the trial.

\section{Statistical methods}

\section{Statistical methods for primary and secondary outcomes}

The primary efficacy analysis will be performed on an intention-to-treat basis. A Cox proportional hazards model will be used for the final analysis of the primary outcome. Continuous parameters will be reported as the median and interquartile range (IQR) and compared with nonparametric tests.

The Hodges-Lehmann estimate will be used to calculate 95\% CIs for the difference between medians. Categorical variables will be reported as counts and percentages and compared with the $\chi 2$ test. We will measure odds ratios for the clinical endpoint with the Mantel-Haenszel test. Kaplan-Meier test will be done to assess the time to clinical deterioration. We will use the log-rank test to compare endpoint-free survival between treatment and placebo group.

Statistical significance will be set at $\mathrm{p}<0.05$, and all tests will be 2-tailed. IBM SPSS statistical software of the latest available version will be used for all statistical analyses. All reporting will adhere to the consolidated standards of reporting trials (CONSORT) guideline. The statistical analysis plans (SAP) providing all details of the analyses will be updated before conducting any data analysis.

\section{Methods for additional analyses (e.g. subgroup analyses)}

Additional analysis will be done for different gender and age subgroups. A cox proportional hazards model will be used for the analysis of the primary endpoints of subgroups. For analysis of the mortality of each of these subgroups, a logistic regression model will be used.

\section{Methods in analysis to handle protocol non-adherence and any statistical methods to handle missing data}

Multiple imputations will be used for missing data. The primary analysis will use the intention-to-treat principle and appropriate statistical analysis will be undertaken to assess the robustness of the findings 


\section{Oversight and monitoring}

Composition of the data monitoring committee, its role and reporting structure

An independent data safety monitoring board (DSMB) will be formed by the ethical review committee of Dhaka Medical College upon request by the principal investigator. It will consist of five members, none directly or indirectly related to this trial. They will have no financial or other conflicts of interest. They will periodically monitor protocol adherence, the safety of the participants will make recommendations to continue or terminate the study or modify sample size.

\section{Adverse event reporting and harms}

Adverse events (SE) and serious adverse events (SAE) will be actively looked for and reported from the time of randomization today 21 . SAEs reported by the participants after day 21 will be recorded if investigators determine that these SAEs are related to the study drugs. SAEs will be followed up until the SAE has subsided. Investigators are responsible for collecting, assessing, reporting, and managing AEs. AEs will be fully investigated and recorded in the CRF, including onset date, the duration of $\mathrm{AE}$, symptoms/signs, severity, action taken to manage the $\mathrm{AE}$ and the outcome of the $\mathrm{AE}$.

\section{DISCUSSION}

This is the first study in Bangladesh to observe the efficacy of colchicine in moderate symptomatic COVID-19 patients. Conducting a double-blind, placebo-controlled, randomized clinical trial during a pandemic is challenging. Limitation of resources and lack of proper funding increases this challenge more. Getting a large number of participants from the hospitalized moderate symptomatic patient is also a challenge because, in our settings, only severe symptomatic patients usually got admitted into hospitals.

\section{ACKNOWLEDGEMENTS}

Authors would like to acknowledge the contribution of Incepta Pharmaceuticals Ltd. 40, Shahid Tajuddin Ahmed Sarani, Tejgaon industrial area, Dhaka, Bangladesh, emailinfo@inceptapharma.com, for their kind approval to donate colchicine and placebo for our participants.

Funding: It is an investigators' initiated study. The study drug and placebo comparator is being donated by a local pharmaceutical company, Incepta Pharmaceuticals Ltd. 40, Shahid Tajuddin Ahmed Sarani, Tejgaon industrial area, Dhaka-1208, Bangladesh, emailinfo@inceptapharma.com. But they will not have any role in study design; collection, management, analysis, and interpretation of data; writing of the report; and the decision to submit the report for publication. They will not have any authority over any of these activities
Conflict of interest: None declared

Ethical approval: This trial has been approved by the ethical review board of the Dhaka Medical College, Dhaka, Bangladesh. The memo number is ERCDMC/ECC/2020/128, Dated June 08, 2020

\section{REFERENCES}

1. World health organization. Coronavirus disease (COVID-19) Situation Report-132 Data as received by WHO from national authorities. Available at: https://www.who.int/docs/defaultsource/coronavirus e/situation-reports/20200531-covid-19-sitrep-

132.pdf?sfvrsn=d9c2eaef_2.) Accessed on: 31 May 2020.

2. Saey TH. Repurposed drugs may help scientists fight the new coronavirus. Available at: https://www.sciencenews.org/article/coronaviruscovid19-repurposed-treatments-drugs. Accessed on: 10 March 2020.

3. World health organization. Naming the coronavirus disease (COVID-19) and the virus that causes it. Available at: https://www.who.int/emergencies/ diseases/novel-coronavirus-2019/technicalguidance/naming-the-coronavirus-disease-(COVID2019)-and-the-virus-that-causes-it. Accessed on: 20 April 2020.

4. Ren LL, Wang YM, Wu ZQ, Xiang ZC, Guo L, Xu $\mathrm{T}$, et al. Identification of a novel coronavirus causing severe pneumonia in human: a descriptive study. Chinese Med J. 2020;133(9):1015-24.

5. Focos D, Anderso A, Tang J, Tuccori M. Convalescent plasma therapy for COVID 19. Preprints (www.preprints.org). 2020.

6. lauer SA, Grantz KH, Jones FK. The Incubation Period of Coronavirus Disease 2019 (COVID-19) From Publicly Reported Confirmed Cases: Estimation and Application. Ann Intern Med. 2020;172(9): 577-82.

7. Liu Z, Chu R, Gong L, Su B, Wu J. The assessment of transmission efficiency and latent infection period in asymptomatic carriers of SARS-CoV-2 infection. Int J Infect Dis. 2020;99:325-327.

8. Antonio V, Francesco F, Chiara P, Giovanni G.Cytokine storm and colchicine potential role fighting SARS-CoV-2 pneumonia. Italian J Med. 2020;14(2).

9. Zaim S, Chong JH, Sankaranarayanan V, Harky A. COVID-19 and Multiorgan Response. Curr Probl Cardiol. 2020;45(8):100618.

10. Baraboutis IG, Gargalianos P, Aggelonidou E. Initial Real-Life Experience from a Designated COVID-19 Centre in Athens, Greece: a Proposed Therapeutic Algorithm. SN Compr Clin Med. 2020;2:689-93.

11. Jamilloux Y, Henry T, Belot A, Viel S, Fauter M, El Jammal T, Walzer T, François B, Sève P. Should we stimulate or suppress immune responses in COVID19? Cytokine and anti-cytokine interventions. Autoimmun Rev. 2020;19(7):102567. 
12. Zhang W, Zhao Y, Zhang F. The use of antiinflammatory drugs in the treatment of people with severe coronavirus disease 2019 (COVID-19): The Perspectives of clinical immunologists from China. Clin Immunol. 2020;214:108393.

13. Soy M, Keser G, Atagündüz P, Tabak F, Atagündüz I, Kayhan S. Cytokine storm in COVID-19: pathogenesis and overview of anti-inflammatory agents used in treatment. Clin Rheumatol. 2020;39(7):2085-94.

14. Deftereos S, Giannopoulos G, Vrachatis DA, Siasos G, Giotaki SG, Cleman M, et al. Colchicine as a potent anti-inflammatory treatment in COVID-19: can we teach an old dog new tricks? Eur Heart J Cardiovasc Pharmacother. 2020;6(4):255.

15. Deftereos SG, Giannopoulos G, Vrachatis DA. Effect of Colchicine vs Standard Care on Cardiac and Inflammatory Biomarkers and Clinical Outcomes in Patients Hospitalized With Coronavirus Disease 2019: The GRECCO-19 Randomized Clinical Trial. JAMA Netw Open. 2020;3(6):e2013136.

16. National Guidelines on Clinical Management of Coronavirus Disease 2019 (COVID-19). Version 7.0 28 May, 2020, DGHS, MOHFW, Government of the People's Republic of Bangladesh.
17. World health organization. Coronavirus disease (COVID-2019) R\&D. Available at: https://www.who.int/blueprint/priority-diseases/ keyaction/novel-coronavirus/en/. Accessed on: 25 March 2020.

18. Wang Y, Fan G, Salam A, Horby P, Hayden FG, Chen $\mathrm{C}$, et al. Comparative Effectiveness of Combined Favipiravir and Oseltamivir Therapy Versus Oseltamivir Monotherapy in Critically Ill Patients With Influenza Virus Infection. J Infect Dis. 2020;221(10):1688-98.

19. Cao B, Wang Y, Wen D, et al. A Trial of LopinavirRitonavir in Adults Hospitalized with Severe Covid19. N Engl J Med. 2020;382:1787-99.

20. Sakpal TV. Sample Size Estimation in Clinical Trial. Perspect Clin Res. 2010;1(2):67-9.

Cite this article as: Rahman M, Rahman MM, Datta PK, Islam K, Hasan P, Haque M, et al. Efficacy of colchicine in moderately symptomatic COVID-19 patients: a study protocol for a double-blind, randomized, placebo-controlled trial. Int J Clin Trials 2021;8(1):43-50. 